tete Selbsthilfe. Das Ergebnis war, dass das Medikament besser wirkte als Placebo, die Effekte der Psychotherapie gegenüber Psychotherapie-Kontrollbedingung waren jedoch noch viel deutlicher. Der Grund war aber nicht, dass Psychotherapie besser als Pharmakotherapie abschnitt, sondern dass die Psychotherapie-Kontrollbedingung besonders schlecht abschnitt. Sie war sogar signifikant schlechter als Placebo.

Wäre es nicht auch möglich, etablierte Psychotherapien gegen eine "Schein-Psychotherapie" zu testen - eine Behandlung, bei der man die Patienten in der Illusion wiegt, eine richtige Psychotherapie zu bekommen?

Hegerl: Das ist sehr schwierig, weil auch der Therapeut verblindet werden müsste. In einer richtigen Psychotherapie ist der Therapeut natürlich überzeugt, von dem was er tut, und dieser Enthusiasmus entfaltet auch seine Wirkung. Bei einer Scheinbehandlung weiß er ja, er macht keine richtige Psychotherapie. Und das würde sich wie Mehltau über die ganzen Sitzungen legen. Natürlich könnte man zwei Psychotherapien oder Interventionen miteinander vergleichen, die beide als wirksam angesehen werden und Hoffnung vermitteln, und dann findet man tatsächlich in den meisten Studien keine Unterschiede.

Lässt sich dann überhaupt irgendwie feststellen, wie groß der Placeboeffekt bei einer regulären Psychotherapie ist?

Hegerl: Das ist schon deshalb schwierig, da unklar ist, wie der Placeboeffekt zu definieren ist. Die Psychotherapeuten betrachten ja die Hoffnungsvermittlung und Aktivierung bei der Psychotherapie mit Recht als ihren therapeutischen Beitrag.

Was bedeutet dies für die Planung künftiger Studien?

Hegerl: Zunächst ist klar, dass alle Studien und Metaanalysen zu Psychotherapien bei Depression mit Wartegruppen unbrauchbar sind. Ein solcher Vergleich ist abstrus und das Ergebnis nicht interpretierbar. Auch andere Psychotherapie-Kontrollbedingungen ergeben wenig Sinn, weil damit kein Placebo-, sondern eher ein Noceboeffekt erzielt wird. Sinnvoll wäre es dagegen, zwei als wirksam angesehene und damit hoffnungsvermittelnde Therapien gegeneinander zu testen, also eine optimale Psychotherapie A mit einer optimalen Psychotherapie B. Bei solchen Studien zur Überlegenheit zweier als wirksam angesehener Behandlungen braucht man aber sehr große Fallzahlen.

Müsste das nicht auch in den Leitlinien besser zur Geltung kommen? In der aktualisierten Depressionsleitlinie werden bei leichter Depression eher Psychotherapien und weniger Antidepressiva empfohlen. Begründet wird dies mit dem fehlenden Unterschied zwischen Placebo und Antidepressiva.

Hegerl: Hier lässt sich tatsächlich eine leichte Gewichtung zugunsten der Psychotherapie beobachten, die ich nach meiner Einschätzung der Datenlage und auch unter Berücksichtigung unserer Metaanalyse so nicht vornehmen würde. Viel größere Sorgen macht mir aber, dass viele Patienten mit mittelschweren und schweren Depressionen noch immer nicht suffizient behandelt werden, weder psychotherapeutisch noch medikamentös. Und dass solche Menschen noch immer Probleme haben, einen Termin beim Facharzt oder Psychotherapeuten zu bekommen. Insgesamt müsste sich die Versorgung viel mehr an die Bedürfnisse der Patienten anpassen, es sollte mehr Kriseninterventionen und Selbsthilfe-Tools geben. info

\section{Werden Antidepressiva unterschätzt?}

In direkten Vergleichsstudien schneiden Antidepressiva etwas besser ab als Psychotherapien. Darauf deutet eine Metaanalyse von Depressionsstudien hin, die von Psychiatern und Psychologen um Professor Ulrich Hegerl von der Universität in Leipzig und Dr. Pim Cuijpers von der Universität Amsterdam veröffentlicht wurde. Die Forscher haben sich gezielt auch Studien angeschaut, in denen Psychotherapien direkt mit Antidepressivabehandlungen verglichen wurden. Damit wollten sie unterschiedlich wirkende Placebo- und Noceboeffekte ausschließen. So wird die Wirksamkeit von Antidepressiva in der Regel verblindet gegen Placebo geprüft. Die Patienten wissen also nicht, ob sie eine wirksame Arznei bekommen. Das wird die Hoffnung auf eine Linderung in beiden Gruppen dämpfen, in der Placebogruppe dürfte sie dennoch höher sein als ohne Behandlung, in der Verumgruppe hingegen geringer als bei einer aktiven Therapie. Das, so die Forscher, könnte die Differenzen zwischen Verum und Placebo reduzieren. Bei der Psychotherapie sei das Gegenteil zu beobachten: Eine Verblindung ist nicht möglich, als Kontrollgruppen dienen daher Wartegruppen oder Selbsthilfeansätze. Die Patienten in der Verumgruppe wissen also, dass sie tatsächlich eine Therapie bekommen, zugleich ist den Patienten in der Kontrollgruppe klar, dass sie nicht optimal behandelt werden. Diese sind dann enttäuscht - hier wäre ein Noceboeffekt zu erwarten.

Insgesamt wurden 35 Vergleichsstudien mit zusammen rund $3.700 \mathrm{~Pa}$ tienten analysiert. Neun der Studien enthielten auch eine Placebogruppe. Über alle Studien hinweg zeigte sich dabei nur ein kleiner, nicht signifikanter Vorteil für die Pharmakotherapie. Wurden nur Vergleiche aus Studien mit Placebogruppen berücksichtigt, ergab sich ein minimaler, nicht signifikanter Vorteil für die Psychotherapie: Die Effektstärke (Hedges g) lag bei 0,02, die „Number Needed to Treat" (NNT) bei 83.

Schauten sich die Forscher um Hegerl hingegen ausschließlich die Vergleiche zwischen Psychotherapie und Pharmakotherapie in Studien ohne Placebogruppen an, dann fanden sie einen ebenfalls geringen, wenngleich signifikanten Vorteil zugunsten der Arzneimittel: Die Effektstärke lag bei 0,13, die NNT bei 14 .

mut

Cuijpers $P$ et al. The effects of blinding on the outcomes of psychotherapy and pharmacotherapy for adult depression: A meta-analysis. European Psychiatry 2015; 30 (6): 685 - 93

\section{Wie könnte man die Versorgung verbessern?}

Hegerl: Vor allem bräuchten wir mehr Psychiater, die ja nach den Hausärzten den größten Beitrag zur ambulanten Versorgung depressiv Erkrankter leisten. Zudem müssen die Möglichkeiten der Selbsthilfe, die sich durch die digitalen Medien ergeben, systematischer genutzt werden. Wir bieten über die Stiftung Deutsche Depressionshilfe das iFightDepression-Tool an, ein internetbasiertes Selbstmanagement-Instrument, das wir zusammen mit europäischen Partnern entwickelt haben. Es ist angelehnt an die Prinzipien der kognitiven Verhaltenstherapie. Dieses Instrument kann von geschulten Hausärzten Patienten mit leichteren Depressionen angeboten werden. Über solche Wege lässt sich das Versorgungsdefizit bei der Psychotherapie etwas lindern, wobei wir aber das Instrument nicht als Ersatz für eine richtige Behandlung ansehen. Bei der breiteren Implementierung des Tools in Deutschland werden wir übrigens von der Deutsche Bahn Stiftung unterstützt, die seit 2014 mit der Stiftung Deutsche Depressionshilfe kooperiert.

Das Interview führte Thomas Müller, Springer Medizin. 